\title{
On the critical free-surface flow over localised topography
}

\author{
J. S. Keeler ${ }^{1,2} \dagger$, B. J. Binder ${ }^{2}$ and M. G. Blyth ${ }^{1}$ \\ ${ }^{1}$ Department of Mathematics, University of East Anglia, Norwich, NR4 7TJ, UK \\ ${ }^{2}$ Department of Mathematical Sciences, University of Adelaide, Adelaide 5005, AUS
}

(Received xx; revised xx; accepted $\mathrm{xx}$ )

Flow over bottom topography at critical Froude number is examined with a focus on steady, forced solitary wave solutions with algebraic decay in the far-field, and their stability. Using the forced Korteweg-de Vries (fKdV) equation the weakly-nonlinear steady solution space is examined in detail for the particular case of a Gaussian dip using a combination of asymptotic analysis and numerical computations. Non-uniqueness is established and a seemingly infinite set of steady solutions is uncovered. Non-uniqueness is also demonstrated for the fully nonlinear problem via boundary-integral calculations. It is shown analytically that critical flow solutions have algebraic decay in the far-field both for the fKdV equation and for the fully nonlinear problem and, moreover, that the leading-order form of the decay is the same in both cases. The linear stability of the steady fKdV solutions is examined via eigenvalue computations and by a numerical study of the initial value $\mathrm{fKdV}$ problem. It is shown that there exists a linearly stable steady solution in which the deflection from the otherwise uniform surface level is everywhere negative.

\section{Introduction}

Two-dimensional critical free-surface flow over a bottom topography produces a widerange of interesting and complex wave behaviour. Critical flow occurs when the Froude number based on the depth of water far upstream and dowstream of a localised topography is equal to unity in both cases. The complex flow dynamics which are observed under unsteady conditions are well known, and the generic features include the formation of an undular bore and a periodic wavetrain downstream of a localised obstacle, as well as the recurrent emission of solitons which appear above the obstacle and then propagate upstream (see, for example, Wu 1987; Ee et al. 2010; Grimshaw \& Smyth 1986; Smyth 1987; Grimshaw \& Smyth 1986). Such features may be undesirable in certain situations as the waves which are generated can damage and erode the banks of waterways (e.g. Ellis et al. 2002; Bishop 2004; Bishop \& Chapman 2004). This motivates the further exploration and understanding of this important critical flow problem and, in particular, a consideration of the possible steady solutions and their stability.

In this study we investigate critical free-surface flow over a Gaussian dip topography. Both weakly nonlinear results computed as solutions to the forced Korteweg-de Vries $(\mathrm{fKdV})$ equation, and fully nonlinear results, obtained as solutions to the full water wave problem, are presented. Flow over a rectilinear dip topography has been discussed by Ee et al. (2010) and Keeler (2017) using phase-plane methods. However, such methods are not available in the case of a smoothly-varying topography. We investigate steady solutions, and in particular examine non-uniqueness of steady solutions, for the Gaussian

$\dagger$ Email address for correspondence: j.keeler@uea.ac.uk 

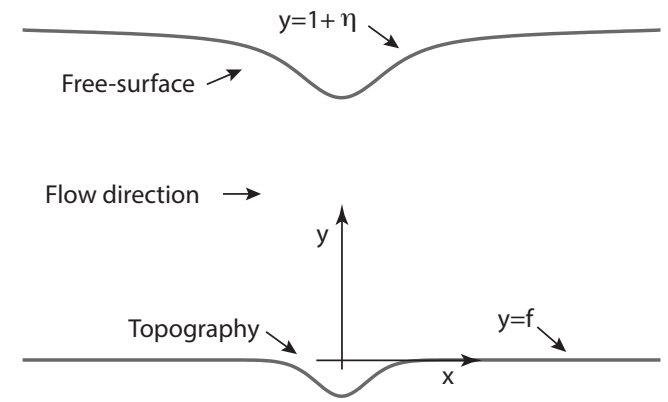

Figure 1.1: Sketch of the non-dimensional flow problem.

topography under critical flow conditions using a combination of analytical and numerical methods. In the context of the fKdV equation, we also discuss the stability of these solutions via eigenvalue computations and by computing time-dependent solutions to the initial value problem.

A sketch of the flow problem is shown in figure 1.1. It is assumed that the flow approaches a uniform stream of constant depth $H$ and speed $U$ as $x^{*} \rightarrow \pm \infty$. The flow is characterised by the dimensionless Froude number

$$
F=\frac{U}{\sqrt{g H}}
$$

which represents the ratio of the characteristic flow speed $U$ to the maximum linear wave speed $\sqrt{g H}$ in a channel of finite depth; critical flow occurs when $F=1$. The free surface is located at $y^{*}=H+\eta^{*}\left(x^{*}, t^{*}\right)$, where $\eta^{*}$ is to be found as part of the solution to the problem. In terms of non-dimensional variables based on the length scale $H$ and time scale $\sqrt{H / g}$, the fKdV equation takes the form (Akylas 1984; Wu 1987)

$$
\eta_{t}+(F-1) \eta_{x}-\frac{3}{2} \eta \eta_{x}-\frac{1}{6} \eta_{x x x}=\frac{1}{2} f_{x}
$$

where $f(x)$ represents a general topographic forcing. We assume that this takes the form of a Gaussian which is symmetric about $x=0$, and, moreover, we seek steady solutions for the free surface shape which possess the same symmetry. More generally, when discussing the stability of these solutions, asymmetric free surface profiles must be allowed for, and to this end we will assume far-field the boundary conditions

$$
\eta, \eta_{x}, \eta_{x x} \rightarrow 0 \quad \text { as } \quad|x| \rightarrow \infty .
$$

As noted above, our focus is on the case $F=1$ for (1.2). For a negative point-force topography corresponding to a delta-function forcing, a phase plane analysis along the lines of Dias \& Vanden-Broeck (2002) can be used to establish a unique steady solution to (1.2), (1.3) which decays algebraically in the far-field. However, in the case of a smoothly varying topography, more than one steady solution is possible. This was demonstrated numerically by Binder et al. (2014). We demonstrate analytically that our steady critical flow solutions decay algebraically in the far-field. We note that in general when $F \neq 1$ exponential far-field decay is expected, as is discussed below.

It is worth emphasising that the results we present for the fKdV equation have wider applicability than to free-surface flows. Indeed, the fKdV equation as been discussed in the context of a wide range of physical scenarios. One application of particular importance, and which is of direct relevance to the present work, concerns internal waves in a stratified flow when the pycnocline, that is the interface between regions of different 
density, as may occur in atmospheric flow over ground relief (see, for example, Baines 1977, 1984; Malanotte-Rizzoli 1984; Grimshaw \& Smyth 1986).

\section{Flow over a Gaussian dip}

A necessary condition for a steady solution at $F=1$ can be found by integrating the steady form of (1.2) and applying the boundary conditions (1.3) to obtain

$$
\int_{-\infty}^{\infty} f(x) \mathrm{d} x \leqslant 0
$$

This shows that there are no steady solutions for non-trivial topography which is everywhere non-negative with $f(x) \geqslant 0$ for all $x$. However, steady solutions may exist for any topography satisfying (2.1) including a Gaussian dip. Henceforth we adopt this form, taking $f(x)=a \exp \left(-b^{2} x^{2}\right)$ for real constants $a<0$ and $b$.

We rewrite the problem in terms of a single parameter by making the change of variables $\eta=\frac{2}{9} b^{2} u$ and $\xi=x b$. Then the steady form of (1.2), after one integration, takes the form

$$
u_{\xi \xi}+u^{2}-\tilde{F} u=\alpha \mathrm{e}^{-\xi^{2}},
$$

where $\alpha=-27 a /\left(2 b^{4}\right)>0$ and $\tilde{F}=\left(6 / b^{2}\right)(F-1)$. We note that in the limit $|\xi| \rightarrow \infty$, wherein $u \rightarrow 0$, there is a balance between the first and third terms on the left hand side of (2.2) producing exponential far-field decay; however, when $F=1$ the balance is between the first and second terms yielding algebraic far-field decay. To summarise we seek solutions to $(2.2)$ in the specific case $F=1$, namely

$$
u_{\xi \xi}+u^{2}=\alpha \mathrm{e}^{-\xi^{2}}
$$

subject to the boundary conditions

$$
u_{\xi}(0)=0, \quad \lim _{\xi \rightarrow \infty} u=0 .
$$

Note that by adopting the former boundary condition we seek only solutions which are even in $\xi$. We begin by examining the extreme cases $\alpha \ll 1$ and $\alpha \gg 1$.

\subsection{The case $\alpha \ll 1$}

Working on the assumption that $\alpha$ is small, we seek an asymptotic solution to (2.3) in the form

$$
u(\xi)=\alpha^{2 / 3} u_{0}(\xi)+\alpha u_{1}(\xi)+\alpha^{4 / 3} u_{2}(\xi)+\cdots
$$

Substituting (2.5) into (2.3) and solving at successive orders of approximation using the first of the conditions in $(2.4)$, we find

$$
u_{0}(\xi)=\bar{u}_{0}, \quad u_{1}(\xi)=\int_{0}^{\xi} \mu(\tau) \mathrm{d} \tau+\bar{u}_{1}, \quad u_{2}(\xi)=-\frac{1}{2} \bar{u}_{0}^{2} \xi^{2}+\bar{u}_{2},
$$

where the $\bar{u}_{i}$ are constants of integration, and $\mu(\tau)=\int_{0}^{\tau} \exp \left(-\tau^{\prime 2}\right) \mathrm{d} \tau^{\prime}$. In the far-field we find that

$$
u(\xi) \sim \alpha^{2 / 3} \bar{u}_{0}+\alpha\left(\frac{\sqrt{\pi}}{2} \xi+\bar{u}_{1}-\frac{1}{2}\right)+O\left(\alpha^{4 / 3}\right)
$$

as $\xi \rightarrow \infty$, which indicates that the expansion will fail when $\xi \sim O\left(\alpha^{-1 / 3}\right)$. Accordingly we introduce an outer region in which the new variable $\zeta=\alpha^{1 / 3} \xi=O(1)$ and posit the 


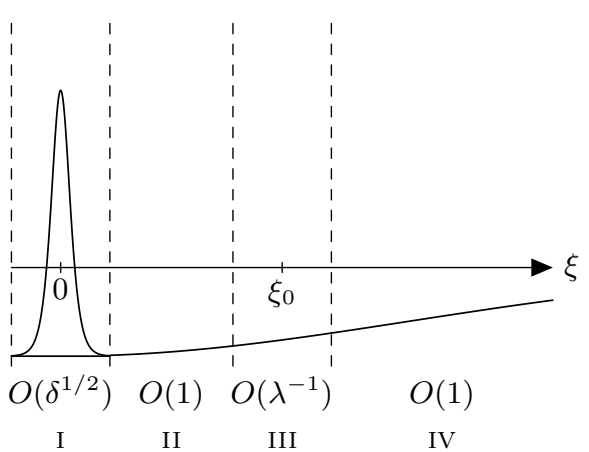

(a)

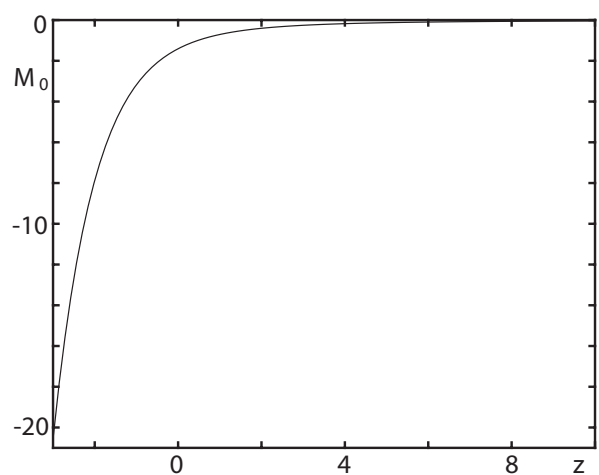

(b)

Figure 2.1: (a) Sketch of the four regions used in the matched asymptotics for large $\alpha$. The decay is exponential in region II and algebraic in region IV. (b) Numerical solution to the region III problem (2.27) subject to (2.28) with the minus sign.

expansion $u=\alpha^{2 / 3} \tilde{u}_{0}(\zeta)+\cdots$ inside this outer region. Substituting into (2.3) we find at leading order and at first order,

$$
\tilde{u}_{0 \zeta \zeta}+\tilde{u}_{0}^{2}=0, \quad \tilde{u}_{1 \zeta \zeta}+2 \tilde{u}_{0} \tilde{u}_{1}=0 .
$$

The solutions which satisfy the second condition in (2.4) are

$$
\tilde{u}_{0}(\zeta)=-\frac{6}{\left(\zeta+d_{1}\right)^{2}}, \quad \tilde{u}_{1}(\zeta)=\frac{d_{2}}{\left(\zeta+d_{1}\right)^{3}},
$$

where $d_{1}, d_{2}$ are constants of integration. Matching to the inner region we find that

$$
\bar{u}_{0}=-\frac{6}{d_{1}^{2}}, \quad d_{1}=2\left(\frac{3}{\sqrt{\pi}}\right)^{1 / 3},
$$

as well as $d_{2}=0$ and $\bar{u}_{1}=1 / 2$. We conclude that for small $\alpha$, there exists a solution with negative elevation at the point of symmetry with $u(0)<0$.

\subsection{The case $\alpha \gg 1$}

In this case it will be convenient to rescale the problem by introducing the new dependent variable $u=\alpha^{1 / 2} U$ so that (2.3) takes the form

$$
\delta U_{\xi \xi}+U^{2}=e^{-\xi^{2}}
$$

where $\delta=\alpha^{-1 / 2}$ and $\delta \ll 1$. Referring to figure 2.1 we see that the asymptotic solution for small $\delta$ has four distinct regions. In the core region, labelled region II in the figure, we expand the solution by writing

$$
U=U_{0}+\delta U_{1}+\delta^{2} U_{2}+\delta^{3} U_{3}+\cdots
$$

Substituting into (2.11) we obtain algebraic equations for the $U_{i}$ at successive orders of approximation which are solved easily. At leading order we find

$$
U_{0}= \pm e^{-\xi^{2} / 2} \text {. }
$$

Discussion of the choice of the sign in $(2.13)$ will be given below. At $O(\delta)$ we have

$$
U_{1}=-\frac{U_{0 \xi \xi}}{2 U_{0}}=\frac{1}{2}\left(1-\xi^{2}\right),
$$


which is valid regardless of the sign taken for $U_{0}$ in (2.13). From the next two orders, we determine the forms

$$
U_{2}= \pm \frac{1}{8}\left(\xi^{4}-2 \xi^{2}-3\right) \mathrm{e}^{\xi^{2} / 2}, \quad U_{3}=\frac{5}{8}\left(\xi^{4}-1\right) \mathrm{e}^{\xi^{2}} .
$$

Although each of the $U_{i}$ in (2.12) satisfies the symmetry condition $U_{i \xi}(0)=0$ required by (2.4), the expansion must nonetheless break down as $\xi \rightarrow 0$ since the first term on the left hand side of (2.11) will become comparable to the second in this limit. Moreover, (2.12) evidently breaks down as $\xi$ increases since successive terms in the expansion grow to become comparable in size to their predecessors. We address the first of these issues first, and consider the solution in the boundary layer at $\xi=0$, namely region I in figure 2.1 .

In region I, we introduce the boundary-layer variable $y=\delta^{-1 / 2} \xi$ and expand the solution by writing

$$
U=\tilde{U}_{0}(y)+\delta \tilde{U}_{1}(y)+\delta^{2} \tilde{U}_{2}(y)+\cdots .
$$

Substituting into (2.11), at leading order we find

$$
\tilde{U}_{0 y y}+\tilde{U}_{0}^{2}=1
$$

subject to the symmetry condition $\tilde{U}_{0 y}(0)=0$ and the condition that $\tilde{U}_{0} \sim \pm 1$ as $y \rightarrow \infty$ to match with region II, the choice of sign in the latter condition being dictated by the choice of sign taken in (2.13). This problem has three possible solutions given by

$$
\text { (i) } \quad \tilde{U}_{0}=1, \quad \text { (ii) } \quad \tilde{U}_{0}=-1, \quad \text { (iii) } \quad \tilde{U}_{0}=-1+3 \operatorname{sech}^{2}(y / \sqrt{2}) .
$$

Referring to the $\left(\tilde{U}_{0}, \tilde{U}_{0 y}\right)$ phase plane, these correspond respectively to fixed points at $\tilde{U}_{0}= \pm 1$, and a homoclinic orbit encircling $\tilde{U}_{0}=1$ which connects $\tilde{U}_{0}=-1$ to itself.

We examine cases $(i)$ and $(i i)$ first. At $O(\delta)$ we have

$$
\tilde{U}_{1 y y} \pm 2 \tilde{U}_{1}=-y^{2} .
$$

The solution which satisfies $\tilde{U}_{1 y}(0)=0$, and which does not grow exponentially as $y \rightarrow \infty$, is given by

$$
\tilde{U}_{1}=\frac{1}{2}\left(1 \mp y^{2}\right)
$$

Since the expressions in (2.20) are forced directly by the solution in the core region II, the matching with that region is immediate. For case $(i i i)$, we have

$$
\tilde{U}_{1 y y}+2\left(3 \operatorname{sech}^{2}(y / \sqrt{2})-1\right) \tilde{U}_{1}=-y^{2} .
$$

By making the change of variables $t=\tanh (y / \sqrt{2})$, this can be transformed into the associated Legendre equation

$$
\frac{\mathrm{d}}{\mathrm{d} t}\left(\left(1-t^{2}\right) \frac{\mathrm{d} \tilde{U}_{1}}{\mathrm{~d} t}\right)+\left(12-\frac{4}{1-t^{2}}\right) \tilde{U}_{1}=-\frac{4 \operatorname{arctanh}^{2} t}{1-t^{2}}
$$

The solution which satisfies the required symmetry condition $\tilde{U}_{1 t}(0)=0$ is given by

$$
\begin{array}{r}
\tilde{U}_{1}(t)=\frac{1}{4} p_{1}(t)\left[\int_{0}^{t} \frac{\operatorname{arctanh}^{2} s}{s^{2}-1} p_{2}(s) \mathrm{d} s-\frac{15}{2} \pi^{2}\right]+\frac{1}{32} p_{2}(t)\left[4 \log \left(1-t^{2}\right)\right. \\
\left.-\log \left(\frac{1+t}{1-t}\right)\left\{\left(1-t^{2}\right) \log \left(\frac{1+t}{1-t}\right)-4 t\right\}-8 \log 2\right],
\end{array}
$$


where

$$
p_{1}(t)=t\left(t^{2}-1\right), \quad p_{2}(t)=15 t\left(t^{2}-1\right) \log \left(\frac{1+t}{1-t}\right)+\frac{50 t^{2}-30 t^{4}-16}{t^{2}-1}
$$

are the homogenous solutions to (2.22). We note that $\tilde{U}_{1}(t) \sim \operatorname{arctanh}^{2} t$ as $t \rightarrow 1$ and so $\tilde{U}_{1} \sim-y^{2} / 2$ ensuring a match with region II.

We now turn our attention to region III. Scrutinising the terms $U_{0}$ and $U_{1}$ in $(2.13)$ and (2.14), we see that the expansion (2.12) in region II breaks down when $\exp \left(-\xi^{2} / 2\right) \sim$ $\frac{1}{2} \delta\left(\xi^{2}-1\right)$. This occurs when $\xi \sim \xi_{0}(\delta)$, where

$$
\xi_{0}=\lambda-\lambda^{-1} \log \left(\lambda^{2} / 2\right)+\cdots,
$$

with $\lambda=(-2 \log \delta)^{1 / 2}$. Inside this narrow region, we introduce the internal layer variable $z=\lambda\left(\xi-\xi_{0}\right)$, and expand by writing

$$
U=\delta \lambda^{2} M_{0}(z)+\delta M_{1}(z)+\cdots
$$

Substituting into equation (2.11), we obtain at leading order,

$$
M_{0 z z}+M_{0}^{2}=e^{-2 z} .
$$

The boundary conditions are

$$
\lim _{z \rightarrow-\infty} M_{0}= \pm \infty, \quad \lim _{z \rightarrow \infty} M_{0}=0 .
$$

Here the $+/-$ sign is required to match to the solution in region II, namely (2.13) with the $+/-$ sign selected respectively. It is possible to compute a solution to this problem numerically for either choice of sign; however, great care is needed when attempting to match the solution with region II, as will be discussed below. We discuss first the case of the minus sign in (2.28). Figure 2.1 shows the result of a calculation using fourth-order Runge-Kutta integration to solve the problem (2.27) with the minus sign chosen in the boundary condition (2.28). An analysis for large negative $z$ reveals that the solution takes the form

$$
M_{0}(z)=-\mathrm{e}^{-z}-\frac{1}{2}+\frac{1}{8} \mathrm{e}^{z}+c_{1} K_{2}\left(2 \sqrt{2} \mathrm{e}^{-z / 2}\right)+\cdots
$$

as $z \rightarrow-\infty$, where $K_{2}$ is a modified Bessel function of the second kind, and $c_{1}$ is a constant which must be determined globally but is not required here. Since it is known that $K_{2}(w) \sim \sqrt{\pi / 2 z} \exp (-2 z) \cdots$ (e.g. Abramowitz \& Stegun 1964), the final term in (2.29) decays superexponentially and matching to region II is possible. For large positive $z$, the forcing term on the right hand side of (2.27) becomes negligible and the dominant balance of the two terms on the left hand side requires the behaviour $M_{0} \sim-6 /\left(z+c_{2}\right)^{2}$, for constant $c_{2}$, as $z \rightarrow \infty$. In region IV, the expansion proceeds as

$$
U(\xi)=\delta \hat{U}_{0}(w)+\cdots
$$

where $w=\xi-\xi_{0}=O(1)$. At leading order we have $\hat{U}_{0 w w}+U_{0}^{2}=0$ and the solution satisfying the far-field condition in (2.4), namely $\hat{U}_{0} \rightarrow 0$ as $w \rightarrow \infty$, is

$$
\hat{U}_{0}=-\frac{6}{\left(w+c_{3}\right)^{2}},
$$

for constant $c_{3}$ which can in principle be found by matching to region III.

As noted above, it is possible to numerically compute a solution to the problem $(2.27)$ subject to (2.28) with the plus sign selected in the boundary condition. However, the numerical solution exhibits small decaying oscillations for large negative $z$. Indeed, in 
the limit $z \rightarrow-\infty$ it can be shown that

$$
M_{0}(z) \sim \mathrm{e}^{-z}-\frac{1}{2}-\frac{1}{8} \mathrm{e}^{z}+\alpha_{1} J_{2}\left(2 \sqrt{2} \mathrm{e}^{-z / 2}\right)+\alpha_{2} Y_{2}\left(2 \sqrt{2} \mathrm{e}^{-z / 2}\right)+\cdots,
$$

for constants $\alpha_{1}, \alpha_{2}$, where $J_{2}$ and $Y_{2}$ are Bessel functions of the first and second kind respectively. As $\xi \rightarrow \infty, J_{2}(\xi) \sim \sqrt{2 / \pi \xi} \cos (\xi-5 \pi / 4)+\cdots$ and $Y_{2}(\xi) \sim \sqrt{2 / \pi \xi} \sin (\xi-$ $5 \pi / 4)+\cdots$ (Abramowitz \& Stegun 1964) and so $M_{0}$ has decaying oscillations as $z \rightarrow-\infty$ in agreement with the numerical solution. Since the solution in region II is non-oscillatory, it is not possible to complete a match between regions II and III, and on this basis we must reject the choice of the plus sign in (2.13) since it does not lead to a consistent asymptotic solution.

We conclude that consistent asymptotic solutions may be constructed for cases (ii) and (iii) in (2.18), but not for case $(i)$. This means that there are two asymptotic solutions consistent with the choice of a minus sign in (2.13), namely cases ( $i i)$ and (iii), but there are no solutions consistent with the choice of a plus sign in (2.13), namely case $(i)$.

\subsection{Numerical results}

In summary, we have found in section 2.2 that it is possible to construct a consistent asymptotic solution using boundary-layer theory for cases (ii) and (iii) in (2.18), but not for case $(i)$. This together with the results of the small $\alpha$ asymptotic analysis in section 2.1 suggests that there exist at least two distinct branches of steady solutions. To carry out a full investigation of the solution space, we solved the steady problem (2.3) numerically employing a shooting technique based on the 4th order Runge-Kutta method. The problem was first reformulated as a first order system in the form $\mathbf{x}_{\xi}=\mathbf{F}(\mathbf{x})$, where $\mathbf{x}=\left(u, u_{\xi}\right)^{T}$ and $\mathbf{F}=\left(u_{\xi}, \alpha s-u^{2}\right)^{T}$. Following the assumed symmetry of the solution, the system was integrated forward from the starting point $\mathbf{x}(\xi=0)=\left(u_{0}, 0\right)^{T}$ for a chosen $u_{0}$. The correct value of $u_{0}$ was determined iteratively until the trajectory of the solution in the $\left(u, u_{\xi}\right)$ phase plane approaches the origin. Formally this should happen in infinite time as $\xi \rightarrow \infty$ but in computational practice the value of $u_{0}$ was refined so that an increasingly large value of $\xi$ was required to reach the origin. There are two qualitatively different ways in which this can happen as follows:

$$
\text { (a) } \frac{u_{\xi}}{u} \sim-\frac{2}{\xi} \quad \text { or } \quad \text { (b) } \frac{u_{\xi}}{u} \sim-2 \xi
$$

as $\xi \rightarrow \infty$. Option (a) represents a balance between the two terms on the left hand side of (2.3) and constitutes algebraic decay of the solution as $-6 \xi^{-2}$ for large $\xi$. In this case the slope of the trajectory tends to 0 - as the origin is approached. Option (b) represents a balance between the first term on the left hand side of (2.3) and the topographic forcing term on the right hand side. In this case the trajectory enters the origin from below with an infinitely negative slope. The computed solution was checked against an independent calculation in which the boundary value problem was solved using a finite difference method on a uniform grid over the truncated domain $[-L, L]$ with $L$ taken to be suitably large. Approximating the second derivative in (2.3) with a centred-difference furnishes a set of nonlinear algebraic equations at each grid point, which were solved using Newton's method.

Figure 2.2 (panels a, b and c) show numerical computations of the two solution types predicted by the asymptotic analysis. We refer to the depression wave in figure $2.2 \mathrm{a}$ as a type I solution and the elevation waves in figure $2.2(\mathrm{~b}, \mathrm{c})$ as type II solutions. The solution space, characterised by the value $u(0)$, is shown in figures $2.2(\mathrm{~h})$ and (i). The predictions of the asymptotic analysis are included in figure 2.2(h) and (i). Numerically, we find that 

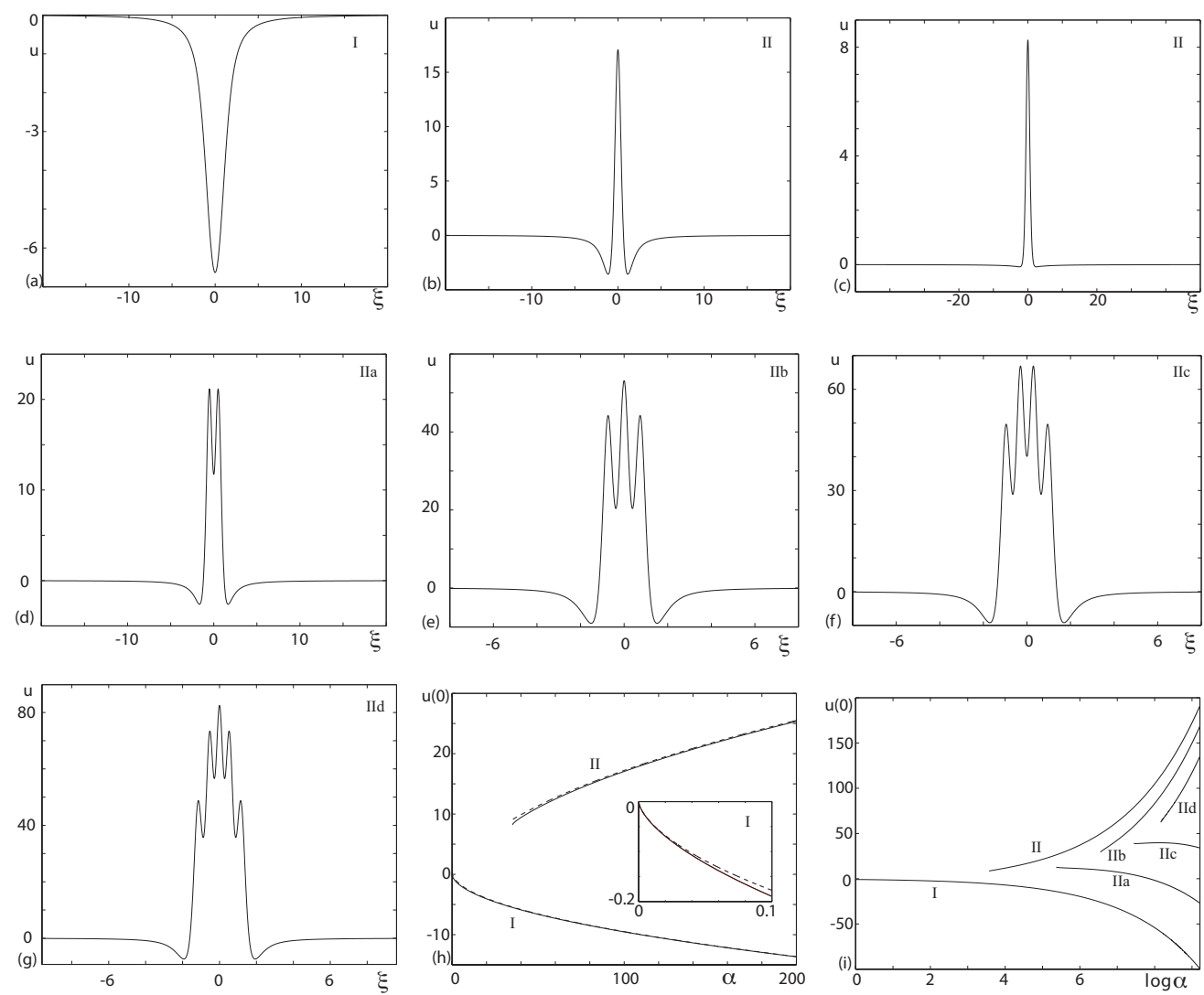

Figure 2.2: (a-g) Free surface profiles: (a) type I at $\alpha=50$; (b) type II at $\alpha=100$; (c) type II at $\alpha=35.2$, close to the branch termination point; (d) type IIa at $\alpha=300$; (e) type IIb at $\alpha=1500$; (f) type IIc at $\alpha=3000$; (g) type IId at $\alpha=5000$. (h) Solution space. The large $\alpha$ behaviour $u(0) \sim-\alpha^{1 / 2}+1 / 2$, case (ii) in (2.18), is shown with a broken curve overlaid on the lower branch. The broken curve overlaid on the upper branch is the large $\alpha$ behaviour for the type II solution $u(0) \sim 2 \alpha^{1 / 2}-4 \log (2)$, case (iii) in (2.18). The inset shows behaviour for small $\alpha$. The broken curve illustrates the small $\alpha$ behaviour $u(0) \sim-\left(6 / d_{1}^{2}\right) \alpha^{2 / 3}+(1 / 2) \alpha$, with $d_{1}$ as given in (2.10). (i) Extended view of the solution space including type IIa, $\mathrm{b}, \mathrm{c}$ and $\mathrm{d}$ solutions. The latter solutions terminate at $\alpha \approx 214.0,699.1,1709.0$ and 3508.0 respectively.

the far-field behaviour (a) in (2.33) is obtained on the lower branch of type I solutions and also on almost all of the upper branch except at the point $\alpha \approx 35.14$, where this branch terminates and where the numerical evidence appears to suggest that behaviour (b) occurs. Further experimentation revealed the existence of a seemingly infinite sequence of new solutions IIa,b,c,d etc., which are not predicted by our asymptotic analysis, and which have oscillations on the free surface localised around the origin. Examples are shown in figure 2.2 (panels $\mathrm{d}$-g). We note the similarity with the solution space over a rectangular trench (Keeler 2017).

\subsection{Fully nonlinear calculations}

We now compare the results obtained for the weakly-nonlinear model to those found for the fully non-linear problem. In the non-dimensionalised steady water-wave equations 

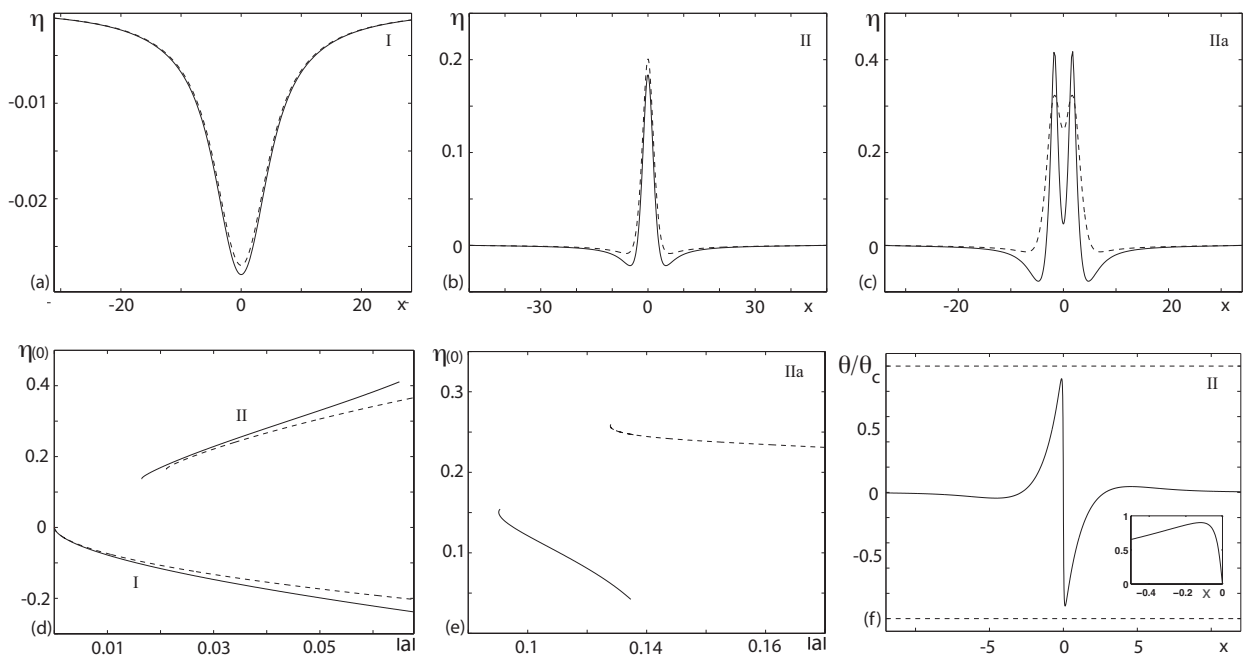

Figure 2.3: Fully nonlinear results for the Gaussian topography (solid curves), $b=0.3$. The broken curves are for the weakly nonlinear solutions. (a) Type I solution, $a=$ -0.0017 . (b) Type II solution, $a=-0.039$. (c) Type IIa solution, $a=-0.134$. (d) Solution space for the type I and II solutions. (e) Solution space for the type IIa solution. (f) Reduced surface angle $\theta / \theta_{c}$, where $\theta_{c}=\pi / 6$, for the highest computed type II wave at $a=-0.06484$. In (f) the inset shows a close-up near to $x=0$.

the velocity potential $\phi(x, y)$ satisfies Laplace's equation throughout the flow domain,

$$
\nabla^{2} \phi=0 \quad \text { in } \quad-\infty<x<\infty, \quad f(x)<y<1+\eta(x) .
$$

On the free-surface the dynamic and kinematic boundary conditions need to be satisfied,

$$
\left.\begin{array}{l}
\frac{1}{2}|\nabla \phi|^{2}+\frac{1}{F^{2}} y=\frac{1}{2}+\frac{1}{F^{2}} \\
\eta_{x} \phi_{x}=\phi_{y}
\end{array}\right\} \quad \text { on } \quad y=1+\eta(x),
$$

along with the no-penetration condition on the bottom,

$$
f_{x} \phi_{x}=\phi_{y} \quad \text { on } \quad y=f(x) .
$$

We solved this system of equations for the unknown functions $\phi(x, y)$ and $\eta(x)$ at $F=1$ numerically for the Gaussian forcing $f(x)=a \exp \left(-b^{2} x^{2}\right)$ with the aim of computing solitary wave solutions. Based on the weakly-nonlinear analysis we would expect the tails of the solitary waves to decay algebraically, and indeed we establish analytically in Appendix A that the full nonlinear solutions decay like $-(4 / 3) x^{-2}$ as $x \rightarrow \pm \infty$, which is precisely the far-field behaviour found for the fKdV equation (2.3). Consequently a large truncation length for the computational domain is required for the computations. The numerical computations were performed first using the boundary integral method described by Tam et al. (2015) over the truncated domain $[-L, L]$ for chosen $L$. Anticipating an inverse square algebraic decay in the far-field, the boundary conditions $\eta_{x} /(1+\eta)=\mp 2 / L$ were applied at $x= \pm L$ respectively. Second, the fully nonlinear system is first reformulated as a single PDE with a non-local term in terms of surface variables alone, as described in Appendix A. The resulting equation (A 11) together with (A 5) is solved with spectral accuracy over the truncated domain $\left[-L^{\prime}, L^{\prime}\right]$, for large $L^{\prime}$ in the mapped domain, assuming periodic boundary conditions, by employing 

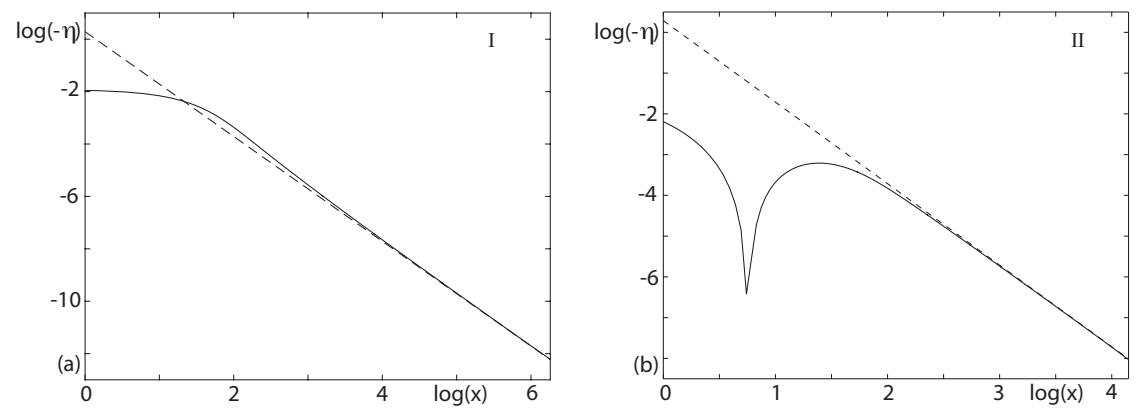

Figure 2.4: Far-field decay of fully nonlinear solutions for a Gaussian topography, $a=$ -0.03 and $b=0.3$, computed on domain $\left[-L^{\prime}, L^{\prime}\right]$ with 2048 collocation points using the method described in Appendix A. Profiles for type I (panel a) and type II (panel b) shown on a log-log plot. In both (a) and (b) the asymptote $\log (-\eta)=\log (4 / 3)-2 \log x$ is shown with a dashed line.

the fast Fourier transform. In the results presented here the computations were done with the first of the numerical approaches, except for the type II branch solutions, where the second approach proved more expeditious particularly in obtaining the steep wave profiles.

The comparison with the weakly nonlinear solutions for the steady fKdV equation (2.3) is shown in figure 2.3(a-e). The type I solution demonstrates a very good agreement with a small discrepancy at the wave minimum. Note that only a portion of the wave is shown around the origin since the two solutions are visually indistinguishable over the remainder of the range. There is very good agreement on the type I solution branch for small $|a|$ as expected. The fully nonlinear type II solution branch exhibits similar behaviour to the weakly nonlinear prediction but while the latter branch continues indefinitely through negative values of $a$, the former terminates at a finite value of $a$. We note that the fully nonlinear branch cannot possibly follow the weakly nonlinear branch for large $a$ since it is clear from the Bernoulli equation in (2.35) that the surface elevation is bounded above with $\eta \leqslant 1 / 2$ in the case $F=1$; equality is obtained at a stagnation-point where the first term in the Bernoulli equation vanishes. Such an eventuality is expected at the crest of a Stokes limiting-configuration, as is found for unforced solitary waves (e.g. Hunter \& Vanden-Broeck 1983). We computed the branch up to $a=-0.06484$ (see figure 2.3d) at which point a very large number of mesh points are required to obtain an accurate solution as the wave profile does indeed seem to be approaching a Stokes-limiting configuration with an interior angle of $120^{\circ}$, and a stagnation point, at the crest. The surface angle $\theta$, defined as the angle between the tangent to the surface and the horizontal, is shown in figure 2.3f for the type II profile obtained at $a=-0.06484$. This particular calculation was performed using the numerical method discussed above with $2^{14}=16,384$ points equally-spaced in the mapped domain with $L^{\prime}=100$. Given the very high resolution required, we did not attempt to move further along the branch toward the Stokes-limiting configuration. Wade et al. (2014) experienced similar numerical difficulties close to the Stokes-limiting configuration for an imposed distribution of pressure on the free surface. In the current work the algebraic far-field decay presents an added difficulty.

As was discussed in section 2.3, the weakly nonlinear branch terminates at its righthand end at a negative value of $a$, as can be seen in figure $2.3 \mathrm{~d}$. We find that the fully nonlinear branch also appears to terminate at its right-hand end. Generally solutions along this fully nonlinear branch decay algebraically as $-(4 / 3) x^{-2}$ and this is confirmed 
in figure 2.4, which demonstrates excellent agreement between the analytic prediction and the numerical computation for a sample case along this branch. It is numerically extremely difficult to identify the shift from algebraic decay to exponential decay (behaviour (b) in (2.33)) which we anticipate will herald the end of the branch as it does for the weakly-nonlinear theory.

The solution type IIa also exists in the fully non-linear solution space but there is a large difference between the fKdV and fully-non-linear solution branches (see figures 2.3c,e) as might be expected since the amplitude $a$ is here rather large. It seems reasonable to suppose that the type IIb,c,... branches are also present in the fully nonlinear regime but since they would likely be quantitatively quite different to their weakly- nonlinear counterparts it may not be straightforward to find them, and we have not attempted to do so. We also note in common with the weakly-nonlinear theory, the type IIa branch terminates at its right-hand end; however as with the type II solutions it appears also to terminate at its other end.

\section{Stability and time-dependent behaviour of solutions}

To conclude our study we examine the stability properties of the steady solutions. We construct conditions for the solutions to be stable using the Hamiltonian argument of Camassa \& Wu (1991) and complement these analytical results with careful numerical computations.

\subsection{Stability Analysis}

The aim is to classify the steady solutions we have computed as being neutrally, spectrally, linearly or formally stable. For a definition of these terms, see for example, Holm et al. (1985); however we will remind the reader of their precise meaning as the analysis proceeds.

Our starting point is the time-dependent fKdV equation (1.2) at the critical condition $F=1$, which is repeated here for convenience,

$$
\eta_{t}-\frac{3}{2} \eta \eta_{x}-\frac{1}{6} \eta_{x x x}=\frac{1}{2} f_{x}(x)
$$

To investigate the stability we write $\eta(x, t)=\eta_{s}(x)+\epsilon \zeta(x, t)$, where $\eta_{s}$ is a steady solution of (3.1) and $\zeta$ is a time-dependent perturbation, and where $\epsilon \ll 1$. We demand that the perturbation $\zeta$ satisfies the same far-field conditions as $\eta$, namely equation (1.3). Substituting into (3.1) and retaining terms at $O(\epsilon)$ we obtain

$$
\zeta_{t}=\mathcal{M} \zeta=-\frac{\partial}{\partial x}(\mathcal{K} \zeta), \quad \mathcal{K}=-\frac{1}{6} \partial_{x x}-\frac{3}{2} \eta_{s}
$$

The general solution to (3.2) may be written in the form (e.g. Chang \& Demekhin 2002 , section 7.3$)$

$$
\zeta(x, t)=\sum_{k=1}^{N_{p}} a_{k} g_{k}(x) \mathrm{e}^{s_{k} t}+\int_{\Gamma} a(s) g(x, s) \mathrm{e}^{s t} \mathrm{~d} s,
$$

where the $a_{k}$ and $a(s)$ are expansion coefficients corresponding respectively to the $N_{p}$ values of $s$ in the point spectrum and to the continuous spectrum of the operator $\mathcal{M}$. These satisfy the relation

$$
\mathcal{M} g=s g
$$

(with $k$ subscripts appended in the case of the point spectrum). The integral in the second 
term in (3.3) is taken over the contour $\Gamma$ which delineates the continuous spectrum in the complex $s$ plane. The eigenfunctions in the point spectrum, $g_{k}(x)$, reside in $L_{2}(-\infty, \infty)$. Furthermore, they satisfy the integral constraint,

$$
\int_{-\infty}^{\infty} g_{k}(x) \mathrm{d} x=0 \quad(s \neq 0),
$$

which is obtained by integrating (3.4) over the real line and using the fact that $g_{k} \rightarrow 0$ as $|x| \rightarrow \infty$ (Camassa \& Wu 1991). Consequently the eigenfunctions $g_{k}$ carry zero excess mass. The functions $g(x, s)$ associated with the continuous spectrum $\Gamma$ are bounded as $|x| \rightarrow \infty$. The continuous spectrum itself is determined by examining the behaviour as $|x| \rightarrow \infty$ (e.g. Sandstede 2002). In this limit, the second term in $\mathcal{K}$ defined in (3.2) can be neglected and (3.2) reduces to $\zeta_{t}-(1 / 6) \zeta_{x x x}=0$; adopting the form $\zeta=\exp (s t) g(x, s)$, we find $g=\exp (\mathrm{i} k x)$ and $s=-\mathrm{i} k^{3} / 6$ for $k \in \mathbb{R}$ so that the continuous spectrum occupies the imaginary axis: $\Gamma=\{\mathrm{i} q: q \in \mathbb{R}\}$.

In the case of type I steady solutions, which have the property that $\eta_{s}(x)<0$ for all $x$, it is straightforward to show that the point spectrum of $\mathcal{M}$ does not include the origin (Keeler 2017). In the case of type II steady solutions, numerically computed spectra are found to not include the origin, as will be discussed below.

If there is no eigenvalue in the spectrum in the right-half complex plane, that is with a positive real part, then $\eta_{s}(x)$ is spectrally stable. If the spectrum is contained along the imaginary axis, then the solution is neutrally stable. The solution is linearly stable if for every $\epsilon>0$ there exists a $\delta>0$ such that if in a suitable norm $\|\zeta(x, 0)\|<\delta$ then $\|\zeta(x, t)\|<\epsilon$ for $t>0$. While linear stability clearly implies spectral stability, the converse is not necessarily true (see Holm et al. (1985) for some examples). As was pointed out by Camassa \& $\mathrm{Wu}(1991)$, for a steady solution $\eta_{s}(x)$ which is even in $x$ (resulting from an even topography), the operator $\mathcal{M}$ is odd in $x$, i.e. $\mathcal{M g}(-x)=-\mathcal{M g}(x)$, which means that for each eigenvalue $s$ satisfying equation (3.4), $-s, s^{*}$ and $-s^{*}$ are also eigenvalues, so that the spectrum has a four-fold symmetry in the complex plane. As a consequence, spectral stability can only occur if all of the eigenvalues lie on the imaginary axis, in which case the solution is neutrally stable. Therefore for the fKdV equation with even topography, neutral stability for even solutions is a necessary and sufficient condition for spectral stability.

We have computed the spectrum of the $\mathcal{M}$ operator numerically on a truncated domain $[-L, L]$ with periodic boundary conditions using a spectral method. Sandstede \& Scheel (2000) showed that calculations performed on a periodic domain faithfully reproduce the point and continuous spectra provided the size of the domain is large enough. Bridges et al. (2002) and Barashenkov \& Zemlyanaya (2000) have noted that numerical algorithms for computing eigenvalues in $\mathrm{KdV}$-type problems can lead to spurious eigenvalues leaving/entering the imaginary axis and posing as unstable/stable modes. With this in mind, we have performed careful convergence tests on our results and, furthermore, we confirm our results by comparison with time-dependent simulations to be discussed in the next subsection. For type I solutions the spectrum is found to be contained on the imaginary axis indicating that the flow is spectrally stable in this case. This conclusion will be confirmed via a formal argument to be presented below. In contrast type II solutions are found numerically to be linearly unstable. In all cases we examined we found that the operator $\mathcal{M}$ has just one unstable eigenvalue $s$ in the righthalf plane (excluding $s^{*}$ ). In fact, this was found to be true for all of the type II and type IIa solutions shown in figure 2.2(h). Table 1 illustrates the convergence of the unstable eigenvalue for a sample type II case as the discretisation level $N$ and the truncation length of the computational domain $L$ are varied (similar results are found for a typical 


\begin{tabular}{cccc}
$N$ & $L$ & $\mathrm{~d} x$ & $s$ \\
\hline 1024 & 100 & 0.195 & $1.9068 \times 10^{-3}+0.09881 \mathrm{i}$ \\
2048 & 200 & 0.195 & $3.5399 \times 10^{-3}-0.09955 \mathrm{i}$ \\
4096 & 400 & 0.195 & $3.5612 \times 10^{-3}-0.09968 \mathrm{i}$ \\
8192 & 800 & 0.195 & $3.5591 \times 10^{-3}-0.09967 \mathrm{i}$ \\
8192 & 400 & 0.098 & $3.5612 \times 10^{-3}-0.09968 \mathrm{i}$
\end{tabular}

Table 1: Type II solution at $a=-0.1357, b=0.3(\alpha=226.1)$. Convergence of the eigenvalues in the discrete $\mathcal{M}$ spectrum computed on a computational domain $[-L, L]$ with $N$ equally-spaced points. One value of $s$ in the $\left(s,-s, s^{*},-s^{*}\right)$ quartet is shown for various $N$ and $L$ (and grid size $\mathrm{d} x=2 L / N$ ).
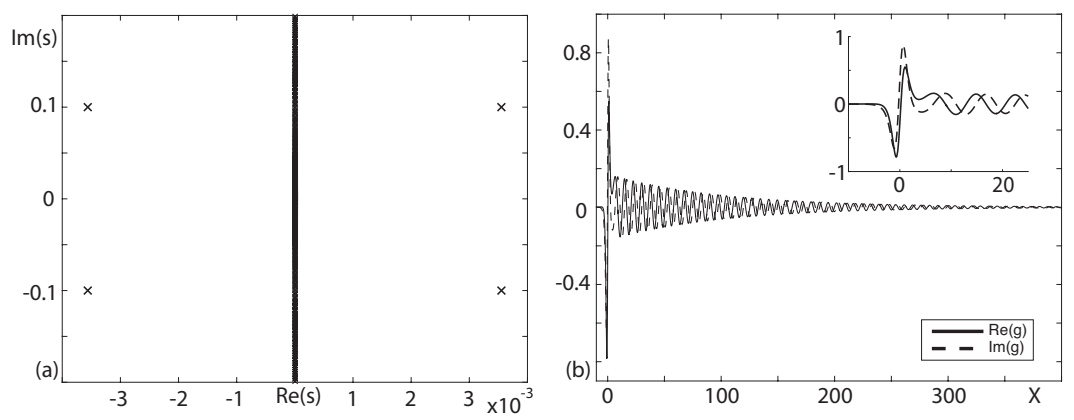

Figure 3.1: Stability properties of the type II solution at $a=-0.1357, b=0.3(\alpha=226.1)$ : (a) Spectrum of the $\mathcal{M}$ operator and (b) the real and imaginary parts of the unstable eigenfunction for $s=0.0036+0.0997 \mathrm{i}$. In (b) the inset shows a close-up of the eigenfunction near to the origin.

IIa case). Evidently convergence is achieved with a rather large number of discretisation points. The spectrum for this case is shown in figure 3.1(a). The unstable eigenfunction is shown in figure $3.1(\mathrm{~b})$. The large $x$ behaviour of the eigenfunction is controlled by the balance $(1 / 6) g_{k}^{\prime \prime \prime} \sim s g_{k}$ in (3.4). Therefore the decay is exponential with $g_{k} \sim \exp (\mu x)$ as $|x| \rightarrow \infty$, where $\mu=(6 s)^{1 / 3}+2 \mathrm{i} k \pi / 3$ for $k=0,1,2$. For the unstable eigenvalue given in table 1 , we obtain the dominant decay rate $\mu=0.73-0.41$ i for $x \rightarrow-\infty$ and $\mu=-0.01+0.84 \mathrm{i}$ as $x \rightarrow \infty$. The latter value indicates a very slow decay downstream requiring a large computational domain. This explains the large value of $L$ required to achieve numerical convergence (see table 1).

As noted above, in the case of a neutrally stable steady solution to the present problem, all of the eigenvalues in the spectrum are necessarily located on the imaginary axis. Linear instability remains a possibility, however. For example, it may be the case that there exist non-simple point-spectrum eigenvalues embedded in the continuous spectrum on the imaginary axis which may lead to algebraic growth in time (Holm et al. 1985). To proceed we adopt an alternative approach suggested by Camassa \& Wu (1991), Chardard et al. (2011) and others, which exploits the fact that (3.1) has a Hamiltonian structure. 
In fact we can write it as

$$
\eta_{t}=-\frac{1}{6} \frac{\partial}{\partial x}\left(\frac{\delta \mathcal{H}}{\delta \eta}\right), \quad \mathcal{H}\left(\eta, \eta_{x}\right)=\frac{1}{2} \int_{-\infty}^{\infty} \eta_{x}^{2}-3 \eta^{3}-6 f \eta \mathrm{d} x,
$$

where $\mathcal{H}$ is the Hamiltonian. Consequently a steady solution $\eta_{s}$ of (3.1) corresponds to a solution of the equation $\delta \mathcal{H} / \delta \eta=0$. With a general perturbation $\zeta$ as defined above, i.e. as $\eta=\eta_{s}+\epsilon \zeta(x, t)$, the second variation of $\mathcal{H}$ at $\eta_{s}$ is given by

$$
\delta^{2} \mathcal{H}=\frac{1}{2} \int_{-\infty}^{\infty} \zeta_{x}^{2}-9 \eta_{s} \zeta^{2} \mathrm{~d} x=\frac{1}{3}\langle\zeta, \mathcal{K} \zeta\rangle, \quad\langle f, g\rangle=\int_{-\infty}^{\infty} f g^{*} \mathrm{~d} x .
$$

If the second variation $\delta^{2} \mathcal{H}$ is positive (or negative) definite then the steady solution $\eta_{s}$ is said to be formally stable. Formal stability implies linear stability, although the converse is not necessarily true (see Holm et al. 1985). Linear instability can therefore be ruled out if $\delta^{2} \mathcal{H}$ is definite.

Since $\mathcal{K}$ is a self-adjoint Schrödinger operator, computing its eigenvalue spectrum constitutes a singular Sturm-Louiville problem on the real line (e.g. Titchmarsh 1962; Coddington \& Levinson 1955) and is a well-studied problem in quantum mechanics and inverse scattering theory (e.g. Landau \& Lifshitz 1977; Drazin \& Johnson 1989). Restricting the admissible perturbations $\zeta$ to the class of $L_{2}(-\infty, \infty)$ square-integrable functions, we obtain the lower bound (Camassa \& Wu 1991; Keeler 2017),

$$
\delta^{2} \mathcal{H} \geqslant \frac{1}{3} \nu_{0}\langle\zeta, \zeta\rangle
$$

Since $\nu_{0}>0$, we can conclude from (3.8) that $\delta^{2} \mathcal{H}$ is positive definite. This means type I solutions (for the trench and Gaussian dip), which are negative definite, are formally stable and hence linearly stable. For our other solution types, $\delta^{2} \mathcal{H}$ is difficult to calculate analytically and instead we used the numerical pseudo-spectral method to determine the $\mathcal{K}$ spectrum and, hence, to calculate $\nu_{0}$. (It should be emphasised that if for a given solution it is found that $\nu_{0}<0$, so that the solution is formally unstable, this does not imply that it is linearly unstable and further investigation is needed.) For the type I solutions we confirmed numerically that $\nu_{0}>0$ implying linear stability in agreement with the calculations of the $\mathcal{M}$ spectrum discussed above. For the type II and IIa solutions we found that $\nu_{0}<0$; as we have already discussed, these solutions are found to be unstable when considering the spectrum of the $\mathcal{M}$ operator.

In the next subsection we perform time-dependent simulations of the unsteady fKdV equation with the goals of confirming the stability properties of the type I and II solutions, and of following the development of the free surface in each case to large time.

\subsection{Time-dependent simulations}

We carried out time-dependent simulations of (1.2) on a truncated spatial domain $[-L, L]$, for a specified value of $L$, assuming periodic boundary conditions and using a method similar to that adopted by Chardard et al. (2011) and Wade et al. (2014). The method computes spatial derivatives spectrally using a discrete Fourier transform and integrates forward in time using the fourth-order Runge-Kutta algorithm. Further details of the method can be found in (Trefethen 2000). In numerical practice, we may obtain solutions that satisfy the boundary conditions in the far-field by taking $L$ sufficiently large. In practice we found that taking $\Delta t \sim 10^{-2}$ and 512 Fourier modes is sufficient to ensure the accuracy of the computations.

Since we assume periodicity in the computations, any disturbance leaving the domain will appear at the opposite end, and such behaviour is clearly unphysical. To prevent 
this in our computations we introduce a so-called sponge layer (see, for example, Boyd 2000; Alias et al. 2013; Grimshaw \& Maleewong 2016) and in fact solve the equation

$$
\eta_{t}-\frac{3}{2} \eta \eta_{x}-\frac{1}{6} \eta_{x x x}+A(x) \eta=\frac{1}{2} f_{x},
$$

where $A(x)=D\left\{1+\tanh \left(b_{0}\left[x-b_{1}\right]\right)\right\}$ for positive parameters $D, b_{0}$ and $b_{1}$. If we choose $b_{1}$ to be close to $L$, then $A$ is non-zero over a narrow region at the left-hand end of the domain. This has the effect of preventing disturbances leaving the upstream end from entering the downstream end (due to the assumed periodicity) and vice versa.

Multiplying (1.2) by $\eta$ and integrating, after making use of (1.3) we obtain

$$
\frac{\mathrm{d} P}{\mathrm{~d} t}=\int_{-\infty}^{\infty} \eta f_{x} \mathrm{~d} x \equiv W(\eta),
$$

where $P=\int_{-\infty}^{\infty} \eta^{2} \mathrm{~d} x$ is the momentum (see Camassa \& Wu (1991) Appendix C). The expression $W$ is termed the wave resistance coefficient by Camassa \& Wu (1991) and is interpreted physically as a measure of the power being supplied by the forcing (see also $\mathrm{Wu} 1987)$. In the absence of forcing, $P$ is a conserved quantity. In general $P=P(t)$ and so $W \neq 0$; it follows from the integral on the right hand side of (3.10) that for a topography which is even in $x$, as will be assumed in this paper, $\eta(x, t)$ will have a left-right asymmetry about $x=0$. In the presence of forcing it is straightforward to show that the excess mass $Q=\int_{-\infty}^{\infty} \eta \mathrm{d} x$ is a conserved quantity. The Hamiltonian defined in (3.6) provides a second conserved quantity, corresponding to the energy, and both of these can be used as a check on the fidelity of numerical time-dependent simulations of (1.2). A further check can be made by computing $W$ using either of the two alternative expressions given in (3.10) and confirming that the same result is obtained in either case. In the numerical results to be discussed in the next section, both $Q$ and $\mathcal{H}$ were performed in the absence of the sponge layer and it was confirmed that they are conserved.

In the results to be presented below a time-dependent calculation is started using the initial condition $\eta(x, 0)=\eta_{s}+\epsilon \hat{\zeta}$ where $\eta_{s}$ is a chosen steady solution, $\hat{\zeta}$ is a perturbation and $\epsilon$ is taken to be a small number. In all of the calculations to be presented below we took $\epsilon=0.025$. If the numerically computed eigenspectrum of the operator $\mathcal{M}$ revealed a positive largest growth rate, the initial perturbation $\hat{\zeta}=\left(g_{k}+g_{k}^{*}\right) / 2$, with $g_{k}$ the corresponding eigenfunction, was used. Otherwise if the numerically computed spectrum was found to contain only purely imaginary eigenvalues, the initial perturbation $\hat{\zeta}=\eta_{s}$ was used.

We note that for a given initial condition and a particular choice of the topographic forcing $f(x)$, the solution to the fKdV equation (1.2) is known to be unique (Wu 1987). Also, we remark that when presenting unsteady simulations of the fKdV equation for topographic forcing similar to that considered here Wu (1987) and Camassa \& Wu (1991) both solved the so-called regularised form of the fKdV equation (see Benjamin et al. 1972), whose equivalence is assured only in the limit of long-waves.

We focus on the case of Gaussian topography with $f(x)=a \exp \left(-b^{2} x^{2}\right)$. Our first goal is to confirm the stability results of section 3.1. Figure 3.2 shows the results of a numerical simulation starting from a small perturbation of the unique type I steady solution $\eta_{s}$ at $\alpha=141(a=-0.085, b=0.3)$. The free-surface profile $\eta(x, t)$ is shown at different times over a portion of the computational domain $[-L, L]$. We note that the solution in the left side of the domain, which is not shown, remains uniformly zero over the duration of the simulation and that no disturbances are observed to propagate upstream. Also shown in the figure are the time signals of the momentum $P$ and the wave resistance coefficient $W$. The initial condition $\eta(x, 0)=1.025 \eta_{s}$ has momentum $P(0)=5.53 \times 10^{-3}$ which is in 

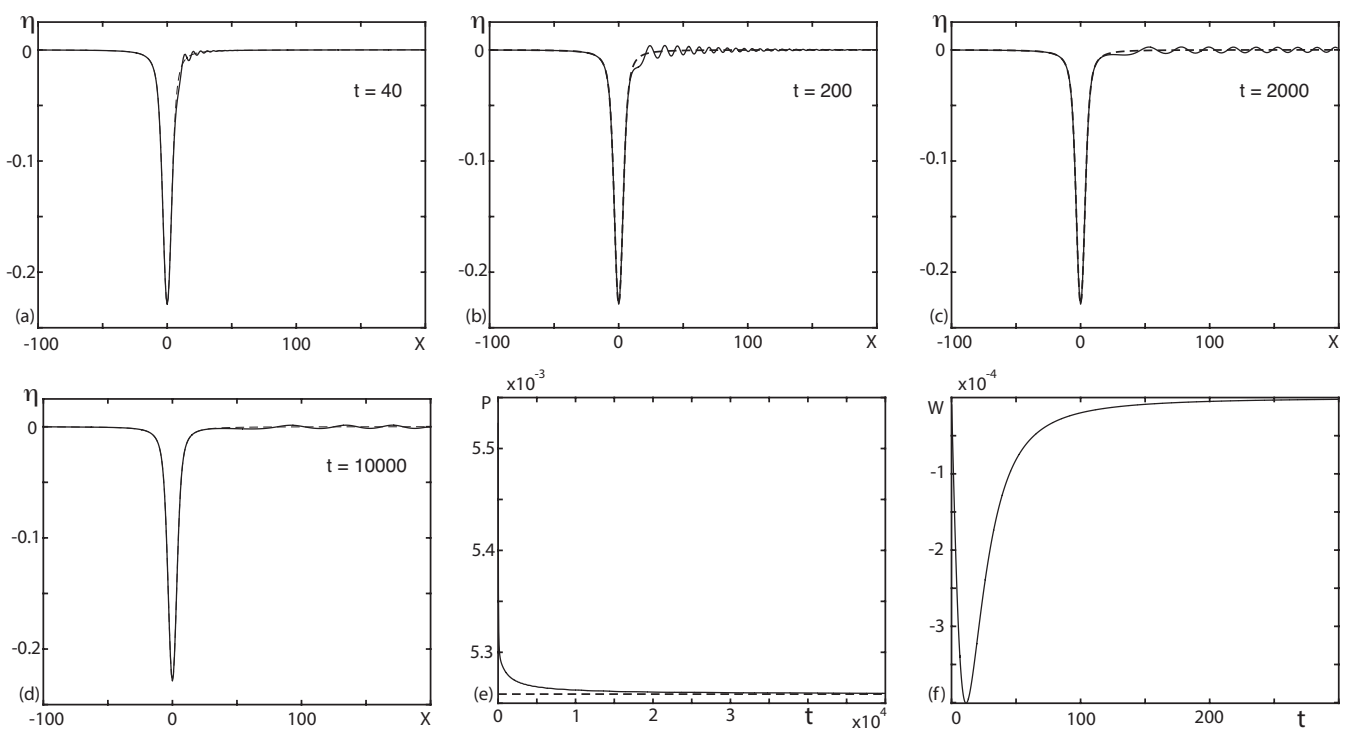

Figure 3.2: Numerical simulation of equation (3.9) with Gaussian forcing for $\alpha=141,(a=$ $-0.085, b=0.3)$ with $L=200$ and $N=512$. The initial condition is $\eta(0, t)=1.025 \eta_{s}$, where $\eta_{s}$ is the unique type I solution at this $\alpha$ (see figure $2.2 \mathrm{~h}$ ). Panels (a-d) show the solution over a portion of the domain at times $t=40,200,2000$ and 10,000. Panels (e) and (f) show the momentum $P$ and $W$ respectively against time. The broken line in panel (e) is the momentum of the type I steady state, $P=5.26 \times 10^{-3}$.

excess of the momentum of the steady state type I solution momentum $P_{s}=5.26 \times 10^{-3}$. The simulation shows that ultimately the system returns to the steady state $\eta_{s}$ as time increases. The wave resistance coefficient $W$ approaches zero at large time, which is a necessary condition for the steady state to be recovered. Also $W$ is negative during the simulation and so, according to (3.10), the momentum $P$ must decrease monotonically. In particular we see that $P \rightarrow P_{s}$ as $t \rightarrow \infty$. A log-log plot of the $W$ signal indicates that $W \sim O\left(t^{-2}\right)$ for large $t$, and so $P$ is expected to approach $P_{s}$ algebraically in time; this explains the very long integration time required to recover the steady state.

We emphasise again that momentum cannot be released from the free surface as long as it is symmetric about $x=0$ since $W$ is zero in this case. Since the initial condition is symmetric, and moreover has a greater momentum than the steady state $\eta_{s}$, it follows that an asymmetric free-surface disturbance must develop in $t>0$ if the steady state is to be recovered in the long term. As can be seen in figure 3.2(a-d), small amplitude waves appear on the leeward side of the free surface. These waves propagate downstream carrying momentum with them. Eventually these waves evanesce and the steady state is recovered. Close inspection of these figures reveals that the free surface, shown with a solid line, overlaps with the steady state $\eta_{s}$, shown with a broken line, over an increasingly wide region as time goes on.

Figure 3.3 shows the results of a numerical simulation starting from a small perturbation of the type II steady solutions $\eta_{s}$ at $\alpha=226.1(a=-0.1357, b=0.3)$ respectively. Very similar results are found for a simulation started from a small perturbation of a type IIa steady solution. The free-surface profile $\eta(x, t)$ is shown at different times over a portion of the computational domain $[-L, L]$. The calculated unstable eigenmode is used as an initial condition for the linearised time-dependent equation (3.2) and excellent agreement is found with the calculated unstable eigenvalue Keeler (2017). The agreement 

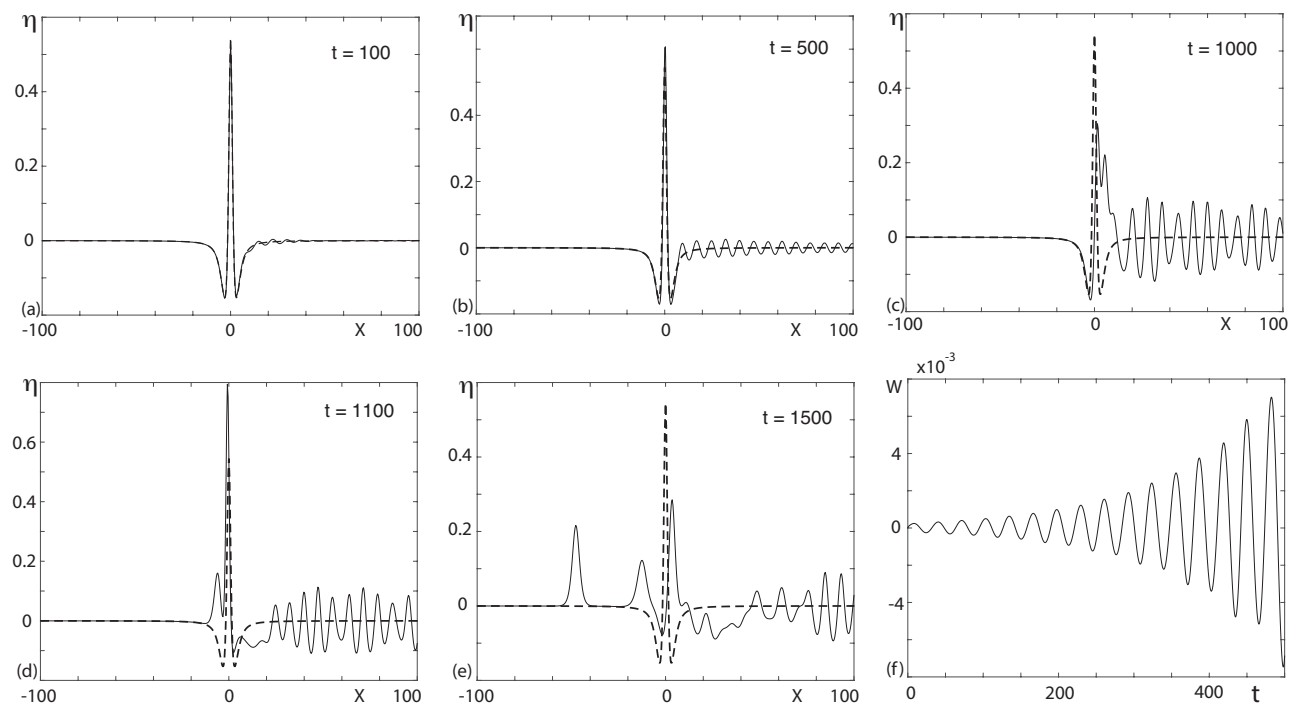

Figure 3.3: Numerical simulation of equation (3.9) with Gaussian forcing for $\alpha=226.1,(a=$ $-0.1357, b=0.3$ ) with $L=400$ and $N=2048$ (note not all of the domain is displayed). The initial condition is $\eta(0, t)=1.025 \eta_{s}$, where $\eta_{s}$ is the unique type II solution at this $\alpha$ (see panel $2.2 \mathrm{~h}$ ). Panels (a-d) show the solution over a portion of the domain at times $t=100, t=500$, $t=1000,1100$ and 1500 . Panel (f) shows $W$ respectively against time. The dotted curve is the steady solution $\eta_{s}$.

for the non-linear time-dependent equation is also good for small times when the initial condition is given by $\eta(x, 0)=\eta_{s}+\frac{1}{2} \epsilon\left(g_{k}+g_{k}^{*}\right)$, with $g$ the unstable eigenmode. The initial condition for the calculations in figure 3.3 is $\eta(x, 0)=1.025 \eta_{s}$ and the different panels show the free-surface profile at different times together with the steady solution, which is shown as a dashed curve. These snapshots show waves travelling downstream in an irregular manner. Additionally, the solution in $x<0$ does not remain uniformly zero over the duration of the simulation and after a finite interval soliton-like disturbances emerge and propagate upstream. To conserve $Q$, the excess mass created by these solitonlike disturbances is balanced by a non- uniform depression region which appears directly downstream of the topography. The time signal of the wave resistance coefficient $W$ is also shown in the figure and is seen to oscillate with increasing amplitude as time increases. We conclude the solution does not return to the steady state as $t$ increases.

We also carried out simulations with the initial condition $\eta(x, 0)=0$. Although a stable steady solution exists in this case, this is not approached at large time. Instead unsteady behaviour is observed whose features are in line with those described by Wu (1987), Camassa \& Wu (1991), Grimshaw \& Smyth (1986) (see in particular section 6 and figure 11), and Grimshaw et al. (2007).

\section{Discussion}

We have examined critical free-surface flow over a localised bottom topography using a weakly-nonlinear forced Korteweg-de Vries model and by way of numerical solutions of the fully nonlinear problem. Critical flow conditions occur when the Froude number is equal to unity both far upstream and far downstream of the localised topography. In the first part of the paper we considered the steady solutions which are found when the topography takes the form of a Gaussian dip (a simple argument dictates that no steady 
solutions are possible in the case of a positive-definite topography). For this topography we studied steady solutions via very carefully conducted numerical calculations. The solution space depends on a single parameter, $\alpha$. For small $\alpha$ there is a unique steady solution, which is negative-definite and which we have termed a type I solution. At a critical value of $\alpha$ a second solution, termed type II, appears which is not singlesigned. At larger values of $\alpha$, further branches of steady solutions appear. In general the steady solutions have algebraic decay in the far-field such that the free surface elevation decays as $-(4 / 3) x^{-2}$ as $x \rightarrow \infty$. Similar algebraic decay can occur for gravity-capillary waves in infinite depth (Akylas et al. 1998) and for gravity-capillary waves in two-layer flow (Sun 1997). To corroborate the numerical solutions, we also constructed asymptotic solutions valid in the limit of small and large $\alpha$. For large $\alpha$ we successfully constructed an asymptotic solution which captures the negative-definite type I solution as well as the type II solution. However, an asymptotic description of the apparently infinite sequence of solution branches, types II a,b,c..., for which an increasing number of ripples are trapped on the free surface around the origin, lies beyond the scope of the present analysis. On this point it is illuminating to highlight the similarity between the presently considered problem and the celebrated Carrier problem discussed at length in, for example, Bender \& Orszag (1999) (p. 464), wherein an increasing number of interior oscillations appear in the solution as the pertinent dimensionless parameter approaches zero. These solutions are not fully captured by a traditional boundary-layer analysis. Determining the asymptotic structure of the type IIa,b,c... solutions in the current work is the subject of our current investigations.

Numerical solutions performed using the boundary integral method and using a conformal mapping technique confirmed the existence of type I, II and IIa solutions in the fully nonlinear regime. However, these solutions do not extend indefinitely to large $\alpha$. Rather, as $\alpha$ is increased, the free surface profiles approach a limiting configuration which is locally similar to that found for a classical Stokes wave with an interior angle of $120^{\circ}$ at the wave maximum.

In the second part of our investigation, we studied the stability of the steady solutions obtained for a Gaussian dip topography first by computing eigenvalues of the linearised system, and then by numerical computation of the full $\mathrm{fKdV}$ initial value problem. For type I solutions all of the eigenvalues were found to lie on the imaginary axis, suggesting linear stability, and this was corroborated by the time-dependent simulations. In fact a simulation initiated with a free-surface which is only a small deviation from a type I solution is seen to slowly relax back to this steady state as time increases; the momentum associated with the initial perturbation is leaked downstream in the form of a wave-train which appears on the downstream side of the free-surface shortly after the start of the simulation. The stability calculations for type II and type IIa steady solutions revealed complex eigenvalues which indicate that these solutions are linearly unstable and this was confirmed by time-dependent simulations initiated using the unstable eigenfunction as the form of perturbation. During these simulations, solitary waves are emitted upstream at regular intervals similar to what has been observed by previous workers. Interestingly, a simulation started from a flat free-surface does not approach the stable type I solution but instead behaviour somewhat similar to that found by $\mathrm{Wu}$ (1987) for critical flow over a positive obstacle is observed: key differences, however, are that the solitary waves which are emitted upstream have different heights and the depression region which appears downstream of the topography is undular rather than flat. The case of a positive topography is well studied (e.g. Wu 1987; Grimshaw \& Smyth 1986; Grimshaw et al. 2007) and analytic results have been obtained; however, to our knowledge no such results are available for a negative topography. 
When reaching our conclusions on the stability properties of the steady solutions for a Gaussian topography/free surface, in addition to numerical eigenvalue calculations and time-dependent simulations, we also applied the Hamiltonian theory of Camassa \& Wu (1991) to show analytically that negative-definite type I solutions are linearly stable. This was done by demonstrating that the $\mathcal{K}$ operator has no negative eigenvalues and hence the second variation of the relevant Hamiltonian is positive-definite. Unfortunately for the type II solutions, which are not single-signed, the results of this analysis are inconclusive. Analytic eigenvalue counts which might be used to deduce stability properties in this case have been discussed at some length in the literature. Notably Pelinovsky (2012) and Kapitula \& Stefanov (2014) (see also the earlier work by Chugunova \& Pelinovsky 2010) independently proved a theorem for linearised systems of the form $\partial_{x} \mathcal{L} u=\lambda u$, which relates the number of potentially unstable eigenvalues $\lambda$ to the number of negative eigenvalues of the self-adjoint operator $\mathcal{L}$. Chardard et al. (2011) used these ideas to infer stability properties of steady, table-top solitary wave solutions for supercritical flow. Unfortunately, the theorem cannot be applied in the present case of critical flow, since it requires that the essential spectrum of the $\mathcal{L}$ operator be bounded away from the origin. Here we have $\mathcal{L}=-\mathcal{K}=(1 / 6) \partial_{x x}+(3 / 2) \eta_{s}$ with essential spectrum $[0, \infty)$, which violates this requirement. We have not been able to identify in the literature an eigenvalue count which applies in this case, and this remains an interesting topic for future work.

The work of MGB was supported by the EPSRC under grant EP/K041134/1.

\section{REFERENCES}

Abramowitz, M. \& Stegun, I. A. 1964 Handbook of Mathematical Functions with Formulas, Graphs and Mathematical Tables. US Department of Commerce.

Akylas, T. R. 1984 On the excitation of long nonlinear water waves by a moving pressure distribution. J. Fluid Mech. 141, 455-466.

Akylas, T. R., Dias, F. \& Grimshaw, R. H. J. 1998 The effect of the induced mean flow on solitary waves in deep water. J. Fluid Mech. 355, 317-328.

Alias, A., Grimshaw, R. H. J. \& Khusnutdinova, K. R. 2013 On strongly interacting internal waves in a rotating ocean and coupled Ostrovsky equations. Chaos 23, 023121.

BAines, G. B. 1977 Upstream influence and Long's model in stratified flows. J. Fluid Mech. 82, 147-159.

BAines, G. B. 1984 A unifed description of two-layer flow over topography. J. Fluid Mech. 146, $127-167$.

Barashenkov, I. V. \& Zemlyanaya, E. V. 2000 Oscillatory instabilities of gap solitions: A numerical study. Comput. Phys. Commun. 172, 22-27.

Bender, C. M. \& Orszag, S. A. 1999 Advanced Mathematical Methods for Scientists and Engineers I. Springer-Verlag.

Benjamin, T. B., Bona, J. L. \& Mahony, J. J. 1972 Model equations for long waves in nonlinear dispersive systems. Phil. Trans. R. Soc. Lond. 272 (1220), 47-78.

Binder, B. J., Blyth, M. G. \& Balasuriya, S. 2014 Non-uniqueness of steady free-surface flow at critical Froude number. EPL 105, 44003.

Bishop, M. J. 2004 A posteriori evaluation of strategies of management: The effectiveness of no-wash zones in minimizing the impacts of boat-wash on macrobenthic infauna. Environ. Manage. 34 (1), 140-149.

Bishop, M. J. \& Chapman, M. G. 2004 Managerial decisions as experiments: An opportunity to determine the ecological impact of boat-generated waves on macrobenthic infauna. Estuar. Coast. Shelf Sci. 61 (4), 613-622.

Blyth, M. G. \& PĂRĂU, E. I. 2016 The stability of capillary waves on fluid sheets. J. Fluid Mech. 806, 5-34.

Boyd, J. P. 2000 Chebyshev and Fourier Spectral Methods. Dover Publications. 
Bridges, T. J., Derks, G. \& Gottwald, G. A. 2002 Stability and instability of solitary waves of the fifth-order KdV equation: A numerical framework. Physica D 172, 190-216.

Camassa, R. \& Wu, T. Y-T. 1991 Stability of forced steady solitary waves. Phil. Trans. R. Soc. Lond. 10, 429-466.

Chang, H.-H. \& Demekhin, E. A. 2002 Complex Wave Dynamics on Thin Films. Elsevier.

Chardard, R., Dias, F., Nyguyen, H. Y. \& Vanden-Broeck, J-M. 2011 Stability of some stationary soutions to the forced KdV equation with one or two bumps. J. Eng. Math. 70, 175-189.

Choi, W. \& Camassa, R. 1999 Exact evolution equations for surface waves. J. Eng. Mech. 125 (7), 756-760.

Chugunova, M. \& Pelinovsky, D. 2010 Count of eigenvalues in the generalized eigenvalue problem. J. Math. Phys. 51 (5), 052901.

Coddington, E. A. \& Levinson, N. 1955 Theory of Ordinary Differential Equations. Tata McGraw-Hill Education.

Dias, F. \& Vanden-Broeck, J. M. 2002 Generalised critical free-surface flows. J. Eng. Math. 42, 291-302.

Drazin, P. G. \& Johnson, R. S. 1989 Solitons: An Introduction. Cambridge university press.

Ee, B. K., Grimshaw, R. H. J., Zhang, D.-H. \& Chow, K. W. 2010 Steady transcritical flow over a hole: Parametric map of solutions of the forced Korteweg-de Vries equation. Phys. Fluids 22, 056602.

Ellis, J. T., Sherman, D. J., Bauer, B. O. \& Hart, J. 2002 Assessing the impact of an organic restoration structure on boat wake energy. J. Coast. Res. 36, 256-265.

Grimshaw, R. \& Smyth, N. 1986 Resonant flow of a stratified fluid over topography. J. Fluid Mech. 169, 429-464.

Grimshaw, R. H. J. \& Maleewong, M. 2016 Transcritical flow over two obstacles: Forced Korteweg-de Vries framework. J. Fluid Mech. 809, 918-940.

Grimshaw, R. H. J., Zhang, D-H. \& ChOw, K. W. 2007 Generation of solitary waves by transcritical flow over a step. J. Fluid Mech. 537, 235-254.

Holm, D. D., Marsden, J. E., Ratiu, T. \& Weinstein, A. 1985 Non-linear stability of fluid and plasma equilibria. Phys. Rep. 1 (116).

Hunter, J. K. \& VAnden-Broeck, J.-M. 1983 Accurate computations for steep solitary waves. J. Fluid Mech. 136, 63-71.

Kapitula, T. \& Stefanov, A. 2014 A Hamiltonian-Krein (instability) index theory for solitary waves to KdV-like eigenvalue problems. Stud. Appl. Math. 132 (3), 183-211.

KeEler, J. 2017 Free-surface flow over bottom topography. PhD thesis, University of East Anglia.

Landau, L. D. \& Lifshitz, E. M. 1977 Quantum Mechanics - Non-Relativistic Theory. Pergamon Press.

Lighthill, M. J. 1958 An Introduction to Fourier Analysis and Generalised Functions. Cambridge University Press.

Longuet-Higgins, M. S. \& Fox, M. J. H. 1977 Theory of the almost-highest wave: The inner solution. J. Fluid Mech. 80 (04), 721-741.

Malanotte-Rizzoli, P. 1984 Boundary-forced nonlinear planetary radiation. J. Phys. Ocean. 14 (6), 1032-1046.

Pelinovsky, D. E. 2012 Spectral stability of nonlinear waves in KdV-type evolution equations. arXiv preprint arXiv:1212.3489 .

SAndstede, B. 2002 Stability of travelling waves. Handbook of Dynamical Systems 2, 983-1055.

Sandstede, B. \& Scheel, A. 2000 Absolute and convective instabilities of waves on unbounded and large bounded domains. Physica D 145 (3), 233-277.

Smyth, N. F. 1987 Modulation theory solution for resonant flow over topography. Proc. R. Soc. Lond. 409, 79-97.

Sun, S.-M. 1997 Some analytical properties of capillary-gravity waves in two-fluid flows of infinite depth. Proc. R. Soc. Lond. A 453, 1153-1175.

Tam, A. T., Yu, Z., Kelso, R. M. \& Binder, B. J. 2015 Predicting channel bed topography in hydraulic falls. Phys. Fluids 27, 112-106.

Titchmarsh, E. C. 1962 Eigenfunction Expansions Associated with Second Order Differential Equations. Part one. Oxford University Press. 
Trefethen, L. N. 2000 Spectral Methods in Matlab. Society for Industrial and Applied Mathematics.

Viotti, C., Dutykh, D. \& Dias, F. 2014 The conformal-mapping method for surface gravity waves in the presence of variable bathymetry and mean current. Procedia IUTAM 11, $110-118$.

Wade, S. L., Binder, B. J., Mattner, T. W. \& Denier, J. P. 2014 On the free surface flow of very steep forced solitary waves. J. Fluid Mech. 739, 1-21.

Wu, T. Y-T. 1987 Generation of upstream advancing solitons by moving disturbances. J. Fluid Mech. 184, 75-99.

\section{Appendix A. Algebraic decay of a solitary wave for critical flow}

In this appendix we demonstrate that a solitary wave solution for critical flow, $F=1$, must decay algebraically in the far-field and we determine the leading order form of this decay. To establish this result we start from the formulation of the problem (2.34)-(2.36). It is convenient then to make a conformal transformation $(x, y) \mapsto(\xi, \zeta)$ mapping the physical domain into the infinite strip $-H \leqslant \zeta \leqslant 0$ in the mapped plane, for some chosen $H$.

Working in a manner similar to that adopted by Choi \& Camassa (1999) in their discussion of periodic water waves, we write down the problem determining the conformal mapping as

$$
\begin{aligned}
y_{\zeta \zeta}+y_{\xi \xi}=0, & \text { in }-H \leqslant \zeta \leqslant 0, \\
y=1+s(\xi) & \text { on } \quad \zeta=0, \\
y=b(\xi) & \text { on } \quad \zeta=-H,
\end{aligned}
$$

where $s(\xi)=\eta(x(\xi))$ and $b(\xi)=f(x(\xi))$ describe the free surface elevation and the bottom topography in the mapped plane respectively. The solution is easily obtained by taking a Fourier transform in $\xi$ with wavenumber $k$, and is given by

$$
y=1+\frac{\zeta}{H}+\frac{1}{2 \pi} \int_{-\infty}^{\infty}\left(\hat{s} \frac{\sinh (k[\zeta+H])}{\sinh (k H)}-\hat{b} \frac{\sinh (k \zeta)}{\sinh (k H)}\right) \mathrm{e}^{\mathrm{i} k \xi} \mathrm{d} k,
$$

where a hat denotes a transformed variable, and $\delta(k)$ is the Dirac delta function. Differentiating (A 4) and noting that $x_{\xi}=y_{\zeta}$ according to the Cauchy-Riemann equations, we find that (see also Viotti et al. 2014; Blyth \& Părău 2016)

$$
\left.x_{\xi}\right|_{\zeta=0}=1 / H-T\left(s_{\xi}\right)+S\left(b_{\xi}\right)
$$

where $T$ and $S$ are non-local operators defined by

$$
T[f(\xi)]=\frac{1}{2 H} \int_{-\infty}^{\infty} f\left(\xi^{\prime}\right) \operatorname{coth}\left(\frac{\pi}{2 H}\left(\xi^{\prime}-\xi\right)\right) \mathrm{d} \xi^{\prime},
$$

and

$$
S[f(\xi)]=\frac{1}{2 H} \int_{-\infty}^{\infty} f\left(\xi^{\prime}\right) \tanh \left(\frac{\pi}{2 H}\left(\xi^{\prime}-\xi\right)\right) \mathrm{d} \xi^{\prime} .
$$

We formulate the flow problem in the mapped plane in terms of the mapped streamfunction $\Psi(\xi, \zeta)$, where $\Psi=\psi(x(\xi, \zeta), y(\xi, \zeta))$, and the streamfunction in the physical plane, $\psi$, is defined in the usual way so that $u=\psi_{y}$ and $v=-\psi_{x}$. The problem is

$$
\begin{aligned}
\Psi_{\zeta \zeta}+\Psi_{\xi \xi}=0, & \text { in }-H \leqslant \zeta \leqslant 0, \\
\Psi=1 & \text { on } \quad \zeta=0, \\
\Psi=0 & \text { on } \quad \zeta=-H,
\end{aligned}
$$


where we have assumed a unit flux in the streamwise direction in keeping with the nondimensionalisation introduced in the main part of the paper. The solution is simply $\Psi=\zeta / H$. The Cauchy-Riemann equations immediately yield the relation $\Phi_{\xi}=1 / H$, where $\Phi(\xi, \zeta)$ is the velocity potential in the mapped plane.

At this stage we have satisfied the kinematic conditions on both the bottom topography and on the free surface. The Bernoulli condition on the free surface in the mapped plane demands that

$$
\frac{F^{2}}{2 J}\left(\Phi_{\xi}^{2}+\Psi_{\xi}^{2}\right)+s=\frac{F^{2}}{2}, \quad J=x_{\xi}^{2}+y_{\xi}^{2},
$$

where all terms are evaluated at $\zeta=0$. Taking into account the fact that $s(\xi)$ is a real function, and considering its Taylor series expansion in Fourier space for both positive and negative values of $k$, we may write down the general small $k$ expansion for the surface elevation in Fourier space as

$$
\hat{s}(k) \sim\left(a_{00}+\mathrm{i} a_{01} \operatorname{sgn}(k)\right)+\left(a_{10}|k|+\mathrm{i} a_{11} k\right)+\left(a_{20} k^{2}+\mathrm{i} a_{21} k|k|\right)+\cdots,
$$

where the $a_{i j}$ are all real. A similar expansion can be written down for the bottom topography function; since this function is prescribed the precise form of its expansion coefficients are known in advance.

Keeping in mind the application to a Gaussian topographic forcing $f(x)$ in the physical plane, as is considered in the main part of the paper, we now assume that $b(\xi)$ decays at least exponentially as $\xi \rightarrow \infty$. By examining the form of the small $k$ expansion of the term in large brackets in the integrand of (A 4), and using (A 5), we find that

$$
\left.x_{\xi}\right|_{\zeta=0} \sim \frac{1}{H}+\left(\frac{\mathrm{i} a_{01}}{H}\right) \xi^{-1}-\left(\frac{a_{10}}{\pi H}\right) \xi^{-2}+\cdots
$$

as $\xi \rightarrow \infty$. The asymptotic form (A 13) has been obtained by making use of Theorem 19 on page 52 of Lighthill (1958).

Substituting our previous results into the Bernoulli equation (A 11), and considering the limit of large $\xi$, we obtain

$$
\begin{aligned}
0=\mathrm{i} a_{01}\left(1-F^{2}\right) \xi^{-1}+ & {\left[\left(F^{2}-1\right) \frac{a_{10}}{\pi}-\frac{3}{2} F^{2} a_{01}^{2}\right] \xi^{-2}+} \\
& {\left[6\left(1-F^{2}\right) a_{21}+6 \mathrm{i} \pi F^{2} a_{01}^{3}-F^{2}\left(9 \mathrm{i} a_{10}-2 H^{2}\right) a_{01}\right] \xi^{3}+\cdots }
\end{aligned}
$$

as $\xi \rightarrow \infty$. If $F \neq 1$, as is the case for either subcritical flow or for supercritical flow, we are forced to conclude that $a_{01}=a_{10}=a_{21}=\cdots=0$; in this case the decay is known to be exponential, as is discussed by Longuet-Higgins \& Fox (1977), Hunter \& Vanden-Broeck (1983) and Wade et al. (2014), for example. If $F=1$, however, it is clear from (A 14) that $a_{01}=a_{21}=0$; moreover at $O\left(\xi^{-4}\right)$ we determine that $a_{10}=(4 / 3) \pi H^{2}$. Applying Lighthill's Theorem 19 to (A 12) we conclude that

$$
s \sim-\frac{4}{3}(\xi / H)^{-2}+\cdots
$$

as $\xi \rightarrow \infty$. We note from (A 13) that $x \sim \xi / H+o(\xi)$ as $\xi \rightarrow \infty$ and hence we find that in the case of critical flow, $F=1$, the free surface elevation decays algebraically as $-(4 / 3) x^{-2}$ for large $x$. Interestingly, this is precisely the form of the far-field behaviour predicted by the steady fKdV equation (2.3). We note that the same inverse square rate of algebraic decay has been demonstrated for gravity-capillary waves in infinite depth by Akylas et al. (1998), and for gravity-capillary waves in two-layer flow by Sun (1997), who established the decay rate rigorously using analytical methods. 